\title{
Evolutionary computation for database marketing
}

Received (in revised form): 10th March, 2003

\section{Siddhartha Bhattacharyya}

is an associate professor of Information and Decision Sciences in the College of Business at the University of Illinois, Chicago. His research covers data mining, data warehousing and various aspect of business intelligence. His work appears in a range of academic journals and he is a regular speaker in different academic and industry conferences and forums.

\begin{abstract}
This paper examines the use of evolutionary computation (EC) techiques, like genetic algorithms and genetic programmes, for data mining and, more specifically, to address problems in database marketing. Beginning with a brief introduction to the basics of genetic search, it provides an overview of two general application areas in database marketing where EC offers distinct advantages: the development of models optimised to specific targeting depths, and models that simultaneously optimise on multiple objectives.
\end{abstract}

\section{INTRODUCTION}

There has been a growing interest in evolutionary computation (EC) techniques addressing problems across diverse domains. Techniques like genetic algorithms and genetic programming offer a search approach based loosely on principles of natural selection and biological evolution. They provide a powerful, general purpose search mechanism that has found application in problems ranging from the design of engines and aerospace structures, scheduling of manufacturing operations and timetabling to portfolio and investment management, modelling of economic phenomena, incorporating adaptive mechanisms in autonomous agents and other models and, in general, for obtaining solutions for hard optimisation problems that are not amenable to solution using traditional approaches.

EC approaches have been applied for classification and data mining, and have been noted to offer unique advantages for modelling in database marketing. ${ }^{1-3}$ These advantages stem from the representational flexibility allowed on the form that a model may take, and from the largely open formulation of the search objective (fitness function). As is well known, models may take a variety of forms based on the nature of the problem and available data, the characteristics in a desirable solution and the modelling technique used. Often the model form is dictated by the specific technique used. For example, logistic regression yields a model for the dependent variable that is functionally linear in the set of predictor variables, while CHAID or CART models take the form of decision trees or restricted rule sets. Model representation is crucial since it largely determines the nature of patterns that are discernible from the 
data. Evolutionary search can be usefully applied with a range of representational forms.

The search objective determines the nature of obtained models and performance characteristics of the models. Traditional approaches like, for example, logistic and least squares regression models seek to maximise likelihood or minimise sum-of-squares of errors; decision tree models seek to minimise some measure of 'impurity' at the nodes; others may seek to minimise classification error rates. ${ }^{4}$ In application, developed models are implemented in some business context, and the business objective may differ in varying degrees from the precise objective function of the model development procedure. For example, a predictive model developed for a mailing campaign may be designed for a high response from a mailing to 20 per cent of the customer file; a logistic regression model used for the purpose may not provide the optimal response for the desired file depth since it is geared toward maximising overall likelihood, which, though related, is somewhat different from the business objective. Similarly, when the business problem entails consideration of multiple, potentially conflicting objectives, a model obtained using conventional approaches may provide a trade-off on the objectives which does not best satisfy requirements. EC, with its ability to tailor the search to specific business needs through the flexibility accorded in fitness function formulation, offers notable advantages in such cases.

This paper gives a brief introduction to evolutionary search, highlighting key issues of significance, and then provides an overview of two general application areas in database marketing where EC offers distinct advantages. Detailed descriptions with experimental evaluations on these applications have been reported in the literature. ${ }^{5-7}$ EC in general includes various techniques; this paper considers the search mechanism of genetic algorithms (GA), which also cover its derivative, genetic programming (GP).

\section{GENETIC SEARCH}

\section{Basic operation}

Genetic algorithms provide a stochastic search procedure based on principles of natural genetics and survival of the fittest. They operate through a simulated evolution process on a population of structures that represent candidate solutions in the search space. Evolution occurs through (1) a selection mechanism that implements a survival of the fittest strategy, and (2) genetic recombination of the selected strings to produce 'offspring' for the next generation.

The basic operation of a simple GA is illustrated in Figure 1, where each population carries $\mathrm{N}$ solutions. Each solution is evaluated against a fitness function (the search objective) that assigns a numeric fitness $f_{I}$. The selection operation probabilistically chooses high fitness solutions into a 'mating pool' solutions with higher than average fitness have a higher occurrence in the mating pool, while low fitness solutions may be eliminated from further consideration. Next, pairs of solutions from the mating pool are recombined to form new solutions ('offspring') for the next generation population. Crossover is a recombination operator where offspring are formed by combining parts of the 'parent' solutions. For example, in Figure 1, crossover applied to Solution 1 and Solution 4 yields Offspring 1 and Offspring 2. The mutation operator makes random changes to the offspring and is applied with low probability. The population of new solutions is then again evaluated against the search objective in an iterative search procedure. 


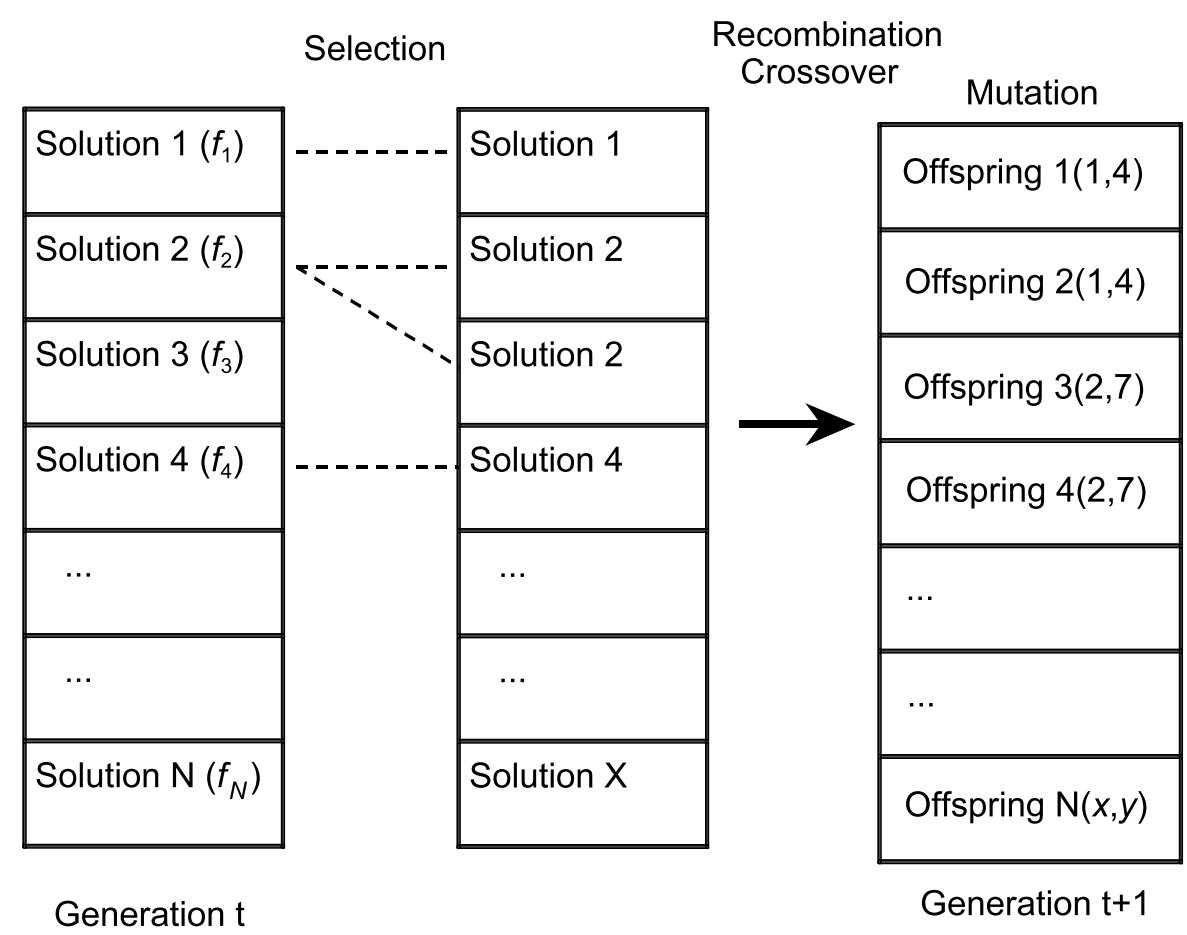

Figure 1: Genetic search - basic operation

Genetic search is known to be effective because of its ability to process and obtain good 'building blocks' (sub-structures in the solution) that progressively yield better solutions, and from the implicit parallelism that arises from its simultaneous consideration of multiple solutions (see Goldberg $^{8}$ for a detailed discussion.) GAs are considered suitable for application to complex search spaces not easily amenable to traditional techniques, and are noted to provide an effective trade-off between exploitation of currently known solutions and a robust exploration of the entire search space. The selection scheme operationalises exploitation and recombination effects exploration.

\section{Representation}

Solutions in a population can take a variety of representational forms, and in a data mining context, the representation determines the nature of patterns that can be discerned from the data. Each population member can specify a weight vector on the predictor variables as in a linear regression; solutions then represent models of the form

$y=1.23 x_{1}+12.8 x_{2}+\ldots$ Solutions can also represent a model expressed in symbolic rule form as in, for example,

$$
[(20 \mathrm{~K}<\text { Income }<=42 \mathrm{~K}) \text { AND }
$$

(Account Balance $<=1.5 \mathrm{~K}$ ) OR $(\ldots) \ldots]=>$ Buyer.

Such representations can capture varied non-linear relationships in data.

The tree-structured representation of genetic programming ${ }^{9}$ allows arbitrary functional forms based on a set of functional primitives (Figure 2). Models here specify a function $f(x)$ of the predictor variables that can be depicted as a parse tree, thus allowing arbitrarily 


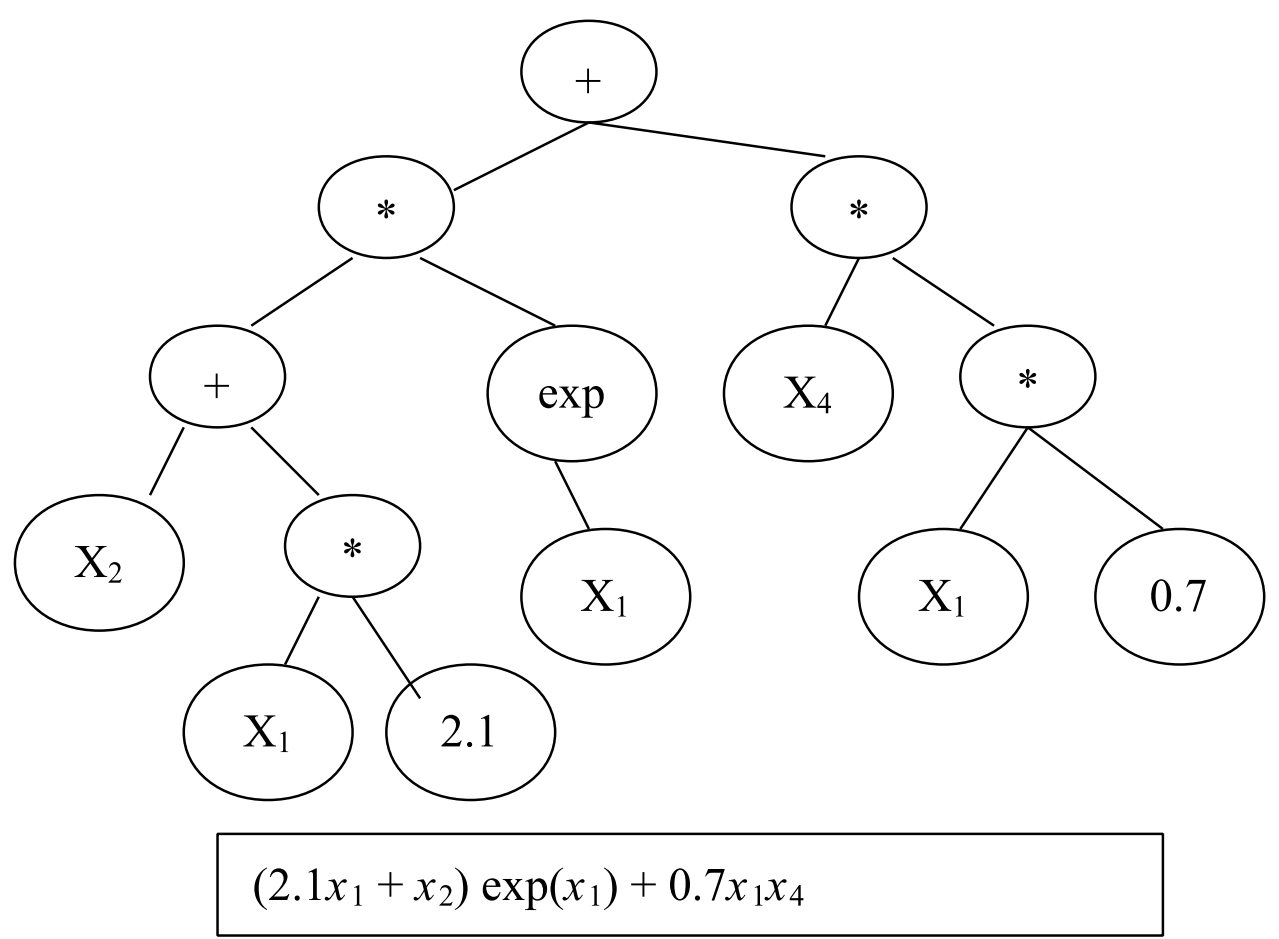

Figure 2: Nonlinear representation of GP

complex functions based on a defined set of primitives.

Various representational forms, suited to specific problems can be used with genetic search. In data mining, a crucial consideration arises from potential overfit problems in developing models from data. Nonlinear representations, while seeking complex patterns, can be prone to capturing relationships that may not generalise well or modelling what may essentially be noise in the data. With adequate controls and with proper model assessment and validation, however, nonlinear representations can provide significant performance benefits.

\section{Fitness function}

The fitness function provides the search objective and provides a numerical figure-of-merit or utility measure for a solution in the population. A key advantage of EC arises from the flexibility allowed in formulation of the fitness function. Unlike in many other techniques, there are no constraints of smoothness, continuity or linearity in the function - the only requirement is that the fitness function provide a numerical value indicating desirability of any solution; it may even be specified as a rule-set for model performance assessment.

For database marketing, the flexibility in fitness function formulation allows the development of models tailored to specific business objectives. For predictive modelling tasks, the dependent variable often determines the search objective. For example, with a binary dependent variable measuring response to some solicitation, or a buy/no-buy decision, etc, the fitness function can be specified to correctly identify 'respondents', or to identify, say, 10 per cent of individuals most likely to buy. Similarly, for a continuous dependent variable, the fitness 
function can be set to obtain a 'profit' model that seeks the most profitable customers, or customers with the highest frequency of response, etc.

The fitness evaluation may also be established to guard against overfit by, for example, utilising resampling techniques. ${ }^{10}$ It can also incorporate a preference for models with fewer variables, or for models exhibiting desired trade-offs among conflicting characteristics. The applications described below utilise such advantages.

The crossover and mutation operators and various other details have been omitted from the brief presentation in this section. Various reference texts provide a detailed discussion of genetic search and related issues. ${ }^{11-13}$

\section{APPLICATIONS IN DATABASE MARKETING}

The general purpose search mechanism of EC can be applied to address a range of problems and issues in data mining and database marketing. Various applications have been reported in the literature. This section provides an overview of two such applications.

\section{Models optimised to targeting depths}

Models, in implementation, are typically used to target only a fraction of individuals in the customer database. For instance, out of a total customer file of a million individuals, resources might permit solicitations to, say, only 200,000 of them. Obviously, the most promising 20 per cent of individuals, as indicated by the model, will be targeted. This targeting depth-of-file may be reflective of resource limitations, may be determined from comparative performance assessment at different file depths, or derived from other considerations. Model performance is typically evaluated at different depths-of-file, using measures like cumulative-lifts or gains from a decile analysis.

This depth-of-file to which model-score ranked individuals are targeted provides potentially useful information for model specification. While the importance of taking this information into account has been noted, ${ }^{14}$ conventional approaches develop models without consideration of how the models will be implemented. An EC-based approach can be used to explicitly take the depth-of-file information into consideration during model development. ${ }^{15,16}$ The essential idea is to obtain models tailored to maximise performance (lifts) at specified depths-of-file. One can thus obtain separate models that optimise performance at different file depths. The development of both 'response models' ${ }^{17}$ and 'profit models" (ie based on binary and continuous dependent variables, respectively) have been reported, and shown to provide significant performance improvements.

Setting up an appropriate fitness function is key to this approach. The fitness function is based on two concerns. While the first focuses on maximising performance at the desired depth-of-file, the second seeks to control overfit to the training data. Focusing exclusively on the first can result in overfitted models that show poor performance on holdout data. Experiments based on real-life data show that a combination of the two fitness concerns yields models with both higher lifts at the considered depths-of-file and which show consistent performance in the training and holdout data. A means for fitness estimation based on resampling has also been developed and noted to yield superior performance (lifts) without the models exhibiting overfit problems. ${ }^{19}$ 
Table 1: Learning with resampling

\begin{tabular}{lllll}
\hline Cumulative lift & First decile & Second decile & Third decile & Fourth decile \\
\hline GA & 284.3 & 182.5 & 168.7 & 145.4 \\
OLS & 194 & 141 & 146 & 133 \\
Improvement over & $46.5 \%$ & $29.4 \%$ & $15.5 \%$ & $9.32 \%$ \\
OLS (\%) & & & & \\
\hline
\end{tabular}

Table $1^{20}$ shows some results from a model developed for a continuous dependent variable measuring profits from a mailing. Here, a linear model representation for a GA was considered, so as to allow for a fair comparison with ordinary least squares (OLS) regression. Performance in terms of cumulative lifts on the holdout data for models optimised for the first, second, third and fourth deciles is shown. The fitness evaluation was based on performance at the specified depths-of-file from multiple bootstrapped samples of the training data. Here, a total of 13,433 observations were randomly split into a training data set of 6,717 observations and a holdout data set of 6,716 observations.

\section{Multi-objective modelling}

Standard modelling approaches embody single performance objectives. Often, however, many real-world problems seek multiple performance measures. Decision makers here desire solutions that simultaneously optimise on multiple objectives. Marketers, for example, may seek individuals who are not only likely to respond to a mailing solicitation, but who also generate high purchase revenues; or, a cellular carrier may seek to identify customers most likely to churn and who also have high usage of services, in order to minimise potential losses from these individuals leaving for a competing offering. Further, different performance objectives can often run counter to each other. Given conflicting objectives, high performance from a model on one objective may correspond to poor performance on the others; a suitable solution here will involve obtaining an acceptable trade-off between the multiple objectives.

A common approach to multicriteria optimisation is to reformulate it as a single objective problem. Linear weighted averages are often considered, with weights on the objectives based on desired trade-offs or other domain knowledge. Where the nature of such trade-offs is not well understood - as is the case in most large data-related scenarios - a precise articulation of preference becomes difficult. Usually, multiple solutions incorporating varying trade-offs among the objectives need to be obtained, and the most satisfactory among these chosen. Other traditional approaches like hierarchical regressions also yield only a single solution, with the model builder having little control over the trade-off manifest in this solution. Multicriteria problems, especially when considering conflicting objectives, do not carry a single best solution, but are instead, characterised by range of solutions, none of which dominate the others with respect to the different objectives. These specify the Pareto frontier of nondominated solutions each of the solutions offers a different level of trade-off, and can be the decision model of choice. This is shown in Figure 3, considering two objectives. Note that solutions that optimise single objectives will be towards the extremities of the frontier and weighted combination of objectives into a single fitness function 


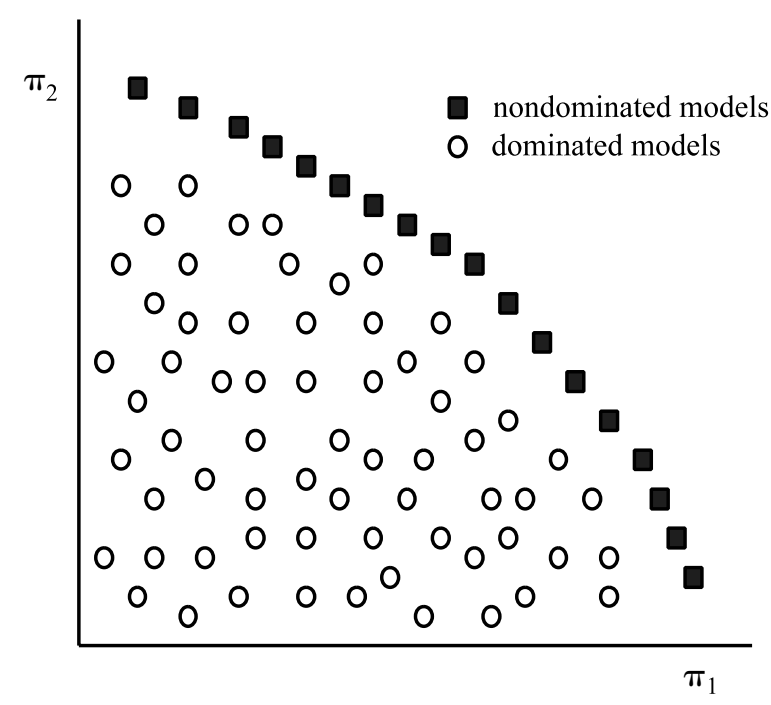

Figure 3: Multiple objectives and nondominated solutions

will foster search towards a specific part of the trade-off frontier only. It is desirable that solutions along the Pareto frontier be examined in determining the most effective model to implement. $A d$ hoc manipulation of the weighted objective function to explore different models is inefficient and tiresome. A preferred approach is to obtain a set of Pareto-optimal solutions in a single invocation of the model development procedure. Evolutionary computation provides such a procedure.

EC-based approaches have been noted to be advantageous for multicriteria optimisation $^{21}$ and their utility in developing multi-objective models for database marketing has been reported. ${ }^{22}$ There, the problem considered was one where a cellular phone provider seeks to identify potential high-value churners so that they can be targeted with some appropriate intervention programme. The specific objective was to identify high-value churners among new installs within the first year of service. Two dependent variables correspond to the two objectives: a binary churn variable indicating a 1 for customers who churned within the first four months and a continuous variable measuring revenue (\$). A linear model representation was considered to allow comparison with logistic (for the churn dependent variable) and OLS regression (for the $\$$ dependent variable), and obtained models were optimised for specified depths-of-file. The fitness function here calculated the fraction of churners and the fraction of revenue obtained by a solution at the specified file depth. A sample data set of 50,000 was randomly separated into equal training and holdout sets of 25,000 observations each, and predictor variables were normalised to zero mean and unit standard deviation.

Figure 4 plots the nondominated solutions for the 10 per cent depth-of-file by performance on the two objectives, churn-lift and \$-lift. Note that the EC-obtained model with best churn-lift performance (rightmost along the horizontal axis) does better on the binary dependent variable than the logistic regression model on that variable. Similarly, the EC-obtained models towards the left end of the trade-off frontier show comparable performance 


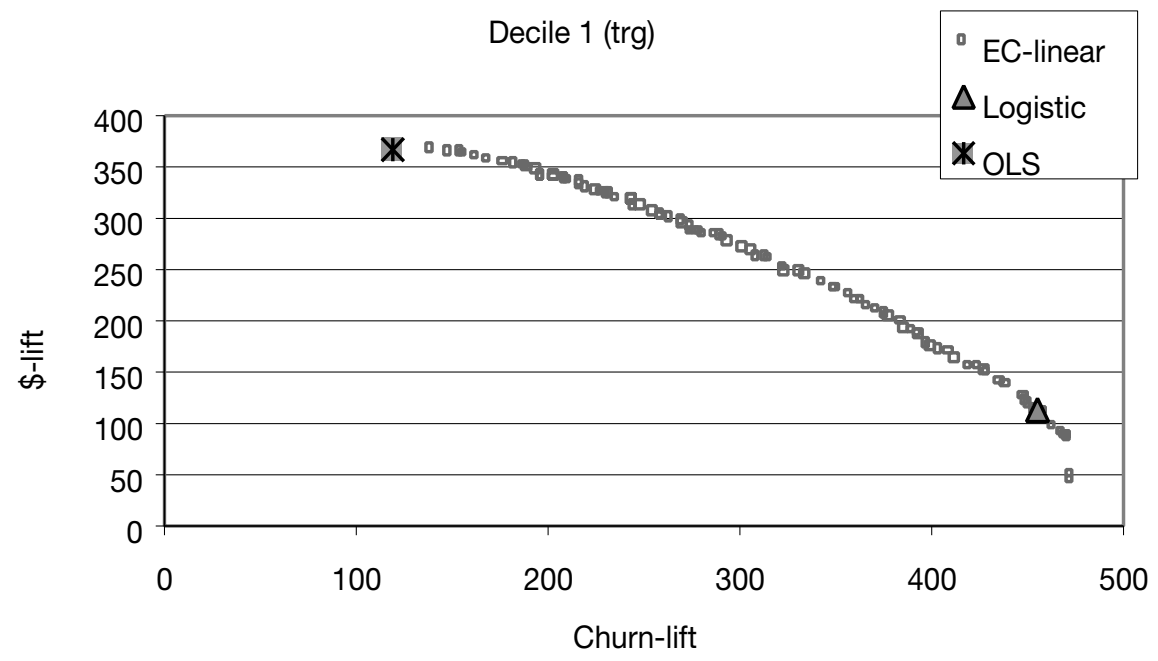

Figure 4: Nondominated solutions for the 10 per cent depth-of-file

Table 2: Range of cum-lifts for models along trade-off frontier (linear EC-based models)

\begin{tabular}{lccccc}
\hline \multicolumn{2}{l}{ Non-dominated models } & Decile 1 & Decile 2 & Decile 3 & Decile 7 \\
\hline Churn-lift & Min, Max & $132.1,464.7$ & $136.0,401.3$ & $119.96,309.8$ & $106.2,139.6$ \\
\$-lift & Min, Max & $46.0,361.5$ & $86.8,271.6$ & $97.4,223.9$ & $114.2,136.6$ \\
\hline
\end{tabular}

on the $\$$-lift to that of the OLS model developed on the $\$$ dependent variable. Similar results were observed for different depths-of-file. Table 2 shows the range of performance (cumulative lifts) of the EC-based models across the trade-off frontier at different file depths.

Given a range of nondominated models, the final selection of model to implement can be based on various issues - for instance, it may be desirable that performance on both objectives be above some minimal threshold level, and judgments may also consider individual, subjective factors. For the churn problem here, an overall modelling consideration can be the maximisation of the expected revenue that can be saved through identification of high-value churners. It can be shown that the product of the churn-lift and \$-lift values gives the cumulative lift on the expected-revenue-saved. A 'best' model can then be one that gives the highest product of lifts. Table 3 shows the performance of the best (highest product-of-lifts) such EC-obtained model. The performance of the logistic and OLS models is also given for comparison. The nonlinear EC-based models were obtained using the tree-structured representation of GP. It

The EC-obtained model giving the highest churn-lift performs at par with the logistic model and the EC-obtained model giving the highest \$-lift performs at par with the OLS model. This is readily observed, for the first decile, from Figure 4 (in fact, the best EC-based model yields higher churn-lift than the logistic model — right extremity of graph.)

Note that instead of considering models optimised for specific depths-of-file, models may be obtained to generally seek good performance across the deciles. This corresponds to a fitness function that measures good 
Table 3: Performance of best model based on product of lifts

\begin{tabular}{llllll}
\hline Performance & & Decile 1 & Decile 2 & Decile 3 & Decile 7 \\
\hline \multirow{2}{*}{ EC-best (linear) } & Churn-lift, \$-lift & $304.9,261.7$ & $265.4,207.4$ & 272.3 .155 .0 & $138.8,126.9$ \\
& Product of lifts & 797.8 & 550.4 & 422.2 & 176.1 \\
EC-best (non-linear) & Churn-lift, \$-lift & $343.7,256.5$ & $343.5,182.1$ & $275.1,178.3$ & $139.4,131.2$ \\
& Product of lifts & 881.5 & 625.5 & 490.4 & 182.9 \\
Logistic regression & Churn-lift, \$-lift & $447.1,111.8$ & $403.4,72.6$ & $295.9,57.4$ & $137.8,66.7$ \\
& Product of lifts & 499.8 & 292.7 & 169.96 & 91.9 \\
OLS regression & Churn-lift, \$-lift & $116.2,360.5$ & $108.1,271.7$ & $99.7,223.2$ & $91.8,136.2$ \\
& Product of lifts & 418.8 & 293.71 & 222.5 & 125.1 \\
\hline
\end{tabular}

Table 4: General fitness function: performance of best models based on product of lift

\begin{tabular}{llllll}
\hline Performance & & Decile 1 & Decile 2 & Decile 3 & Decile 7 \\
\hline \multirow{2}{*}{ EC-best (linear) } & Churn-lift, \$-lift & $303.2,261.7$ & $288.3,188.8$ & 276.7 .151 .3 & $138.1,104.5$ \\
& Product of lifts & 791.4 & 544.3 & 418.6 & 144.3 \\
EC-best (nonlinear) & Churn-lift, \$-lift & $332,252.5$ & $265,223.1$ & $233.9,186.5$ & $132.2,132.1$ \\
& Product of lifts & 838.3 & 591.2 & 436.2 & 176.1 \\
\hline
\end{tabular}

overall lifts-profile on the data. The advantages of the multi-objective EC-based approach described here apply equally for this case too. Table 4 shows the performance of the best of the EC-based models (highest product of lifts at the specific decile levels) developed using such a general fitness function. Observe that the models perform reasonably well across the deciles compared with the decile-optimised models (Table 3); as may be expected, of course, models optimised for specific deciles perform better at that file depth.

\section{CONCLUSION}

Evolutionary computation provides a robust and general purpose search mechanism and holds many advantages for data mining. As evident from the application examples above, a key advantage over traditional modelling techniques comes from their ability to obtain models tailored to specific business needs and contexts. Thus, while traditional techniques may, for example, maximise likelihood or minimise errors, EC-based techniques can obtain models that directly optimise on varied and multiple business objectives. Another advantage arises from the representational flexibility, in being able to explore diverse nonlinear patterns in the data.

In considering their application to direct marketing, model development time and ease of model deployment are important issues to address. Given the iterative nature of the search, EC-based techniques are computer intensive, and as with other computationally intensive techniques applied to data mining problems, call for adequate processing capabilities - most current high-end workstations meet the requirements. Where needed, evolutionary search is also readily parallelisable across multiple processors/machines. The deployment of obtained models depends on the model representation; where models represent some functional relationship among the independent variables, as in the application examples presented in this paper, models can be directly applied to score a customer database (similarly to, for example, regression models.)

This paper has provided an overview of two applications pertaining to model 
development for database marketing. While the presented results were in the context of specific problems, the EC-based procedure for optimisation of lifts presents a general model development approach useful across a range of problems in database marketing. Many applications addressed by database marketing practioners can also profitably utilise the multi-objective modelling approach presented in this paper. Along the lines of the example used here, problems in the telecommunications industry often seek to model customers' tenure in combination with usage identifying people who have long tenure and high usage of services. In catalogue and retail sales, models identifying potential buyers who will not return purchased goods are useful; similarly, models that identify potential respondents to mailings who are also likely to buy some specific product are often sought. Multiple and often conflicting objectives are also seen in the context of many cross-selling marketing campaigns. Further application examples occur in the financial services industry, where, for example, models can seek customers who are likely to be approved for credit and who can also be expected not to make late payments or default on loans.

EC-based approaches have also shown success in other related problems of interest to database marketing. One such problem of large potential impact pertains to exploratory data analysis and variable selection, which, given the typically large data sets in the field, presents one of the more difficult tasks in modelling. Evolutionary search, especially using nonlinear representations as in GP, provides a powerful tool for exploring nonlinear relationships in data. The use of EC for data mining continues to be an area of active investigation. Perhaps the greatest obstacle to their actual use in database marketing to date has been the lack of industry-strength tools providing easy access to evolutionary search facilities in the context of developing models from data, and implementing special features as in, for example, the application examples considered in this paper. ${ }^{23}$

\section{References}

1 Bhattacharyya, S. (1999) 'Direct marketing performance modeling using genetic algorithms', INFORMS Journal of Computing, Vol. 11, No. 13

2 Bhattacharyya, S. (1998) 'Direct marketing response models using genetic search', in 'Proceedings of the Fourth International Conference on Knowledge Discovery and Data Mining (KDD-98)', New York, AAAI Press.

3 Bhattacharyya, S. (2000) 'Evolutionary algorithms in data mining: multi-objective performance modeling for direct marketing', in 'Proceedings of the Sixth International Conference on Knowledge Discovery and Data Mining (KDD-2000)', Boston, AAAI Press.

4 Hand, D., J. (1997) 'Construction and assessment of classification rules', John Wiley and Sons, New York.

5 Bhattacharyya (1999) op. cit.

6 Bhattacharyya (1998) op. cit.

7 Bhattacharyya (2000) op. cit.

8 D. E. Goldberg (1989) 'Genetic algorithms in search, optimization and machine learning', Addison-Wesley, Reading, MA.

9 Koza, J. R. (1993) 'Genetic programming: On the programming of computers by means of natural selection', MIT Press, Cambridge, MA.

10 Bhattacharyya (1999) op. cit.

11 Goldberg (1989) op. cit.

12 Koza (1993) op. cit.

13 Mitchell, M. (1996) 'An introduction to genetic algorithms', MIT Press, Cambridge, MA.

14 Massand, B. and Piatetsky-Shapiro,. G. (1996) 'A comparison of different approaches for maximizing the business payoffs of prediction models', 'Proceedings of the Second International Conference on Knowledge Discovery and Data Mining (KDD-96)', AAAI Press pp. 195-201.

15 Bhattacharyya (1999) op. cit.

16 Bhattacharyya (1998) op. cit.

17 Bhattacharyya (1999) op. cit.

18 Bhattacharyya (1998) op. cit.

19 Bhattacharyya (1999) op. cit.

20 From results in Bhatacharyya (1998) op. cit.

21 Fonseca, C. M. and Fleming, P. J. (1995) 'An overview of evolutionary algorithms in multi-objective optimization', Evolutionary Computation, Vol. 3, No. 1, pp. 1-16.

22 Bhattacharyya (2000) op. cit.

23 The evolveDM software tool provides the capabilities and functionality for the applications described in this paper. 